Monatshefte für Mathematik 80, 257-264 (1975)

(C) by Springer-Verlag 1975

\title{
On a Linear Combination of Some Expressions in the Theory of the Univalent Functions
}

\author{
By \\ Hassoon S. Al-Amiri, Bowling Green, Ohio, \\ and Maxwell O. Reade, Ann Arbar, Mich.
}

(Received 30 April 1974)

Abstract

Let $H(\alpha)$ denote the class of regular functions $f(z)$ normalized so that $f(0)=0$ and $f^{\prime}(0)=1$ and satisfying in the unit disc $E$ the condition

$$
\operatorname{Re}\left\{(1-\alpha) f^{\prime}(z)+\alpha\left(1+z f^{\prime \prime}(z) / f^{\prime}(z)\right)\right\}>0
$$

for fixed $\alpha$. It is known that $H(0)$ is a particular class NW of close-to-convex univalent functions. The authors show the following results: Theorem 1 . Let $f(z) \in H(\alpha)$. Then $f(z) \in \mathrm{NW}$ if $\alpha \leqslant 0$ and $z \in E$. Theorem 2. Let $f(z) \in \mathrm{NW}$. Then $f(z) \in H(\alpha)$ in $|z|=r<r_{\alpha}$ where i) $r_{\alpha}=(1+\sqrt{2 \alpha})^{-1 / 2}, \alpha \geqslant 0$, and ii) $r_{\alpha}=\sqrt{\frac{1-\alpha-\sqrt{\alpha(\alpha-1)}}{1-\alpha}}, \alpha<0$. All results are sharp. Theorem 3. If $f(z)=z+a_{2} z^{2}+a_{3} z^{3}+\ldots$ is in $H(\alpha)$ and if $\mu$ is an arbitrary complex number, then

$$
|1+\alpha|\left|a_{3}-\mu a_{2}^{2}\right| \leqslant(2 / 3) \max [1,|1+2 \alpha-3 / 2 \mu(1+\alpha)|] .
$$

1. Introduction. We consider functions $f(z)$ which are regular in the unit disc $E:|z|<1$ and normalized so that $f(0)=0$ and $f^{\prime}(0)=1$, and we let

$$
I(\alpha, f(z)) \equiv(1-\alpha) f^{\prime}(z)+\alpha\left(1+\left(z f^{\prime \prime}(z) / f^{\prime}(z)\right)\right),
$$

where $\alpha$ is real number. We denote by $H(\alpha)$ the class of functions satisfying $\operatorname{Re}\{I(\alpha, f(z))\}>0$, for fixed $\alpha$ and for all $z \in E$. It is known that $H(0)$ is a particular class NW of close-to-convex univalent functions as demonstrated by K. NosHrRo [2] and S. WARSchaWsKI [4].

In section 2 we show that every $f \in H(\alpha)$ is in NW for $\alpha \leqslant 0$. We are unable to determine the univalency of $H(\alpha)$ if $\alpha>0$. However, in section 3 we obtain the radius of the largest disc $r_{\alpha}$ such that if $f \in \mathrm{NW}$ and $z \in E$, then $f \in H(\alpha)$ for $|z|<r_{\alpha}$. In section 4 some estimates for the coefficients of functions in $H(\alpha)$ are obtained. 
2. The univalency of $H(\alpha)$. We show the following theorem about the univalency of the class $H(\alpha)$.

Theorem 1. Let $f(z) \in H(\alpha)$. Then $f(z)$ is univalent with $\operatorname{Re}\left\{f^{\prime}(z)\right\}>$ $>0, z \in E$, if $\alpha \leqslant 0$ (i. e. $f \in \mathrm{NW})$.

Proof. Let

$$
f^{\prime}(z)=p(z)
$$

To prove Theorem 1 we need to show that if $f \in H(\alpha), \alpha \leqslant 0$, then $\operatorname{Re}\{p(\alpha)\}>0$ in $E$. The case $\alpha=0$ is trivial. If $\alpha<0$, then we assume that $\operatorname{Re}\{p(z)\} \ngtr 0$ in E. Hence, since by $(2), p(0)=1$, then there exists a first $r_{0}$, and $\theta_{0}$ so that $\operatorname{Re}\{p(z)\}>0$ for $|z|<r_{0}$ and

$$
\operatorname{Re}\left\{p\left(z_{0}\right)\right\}=\operatorname{Re}\left\{p\left(r_{0} e^{i \theta_{0}}\right)\right\}=0
$$

where $0<r_{0}<1$. The condition in (3) implies that $\partial \arg p(z) / \partial \theta=0$ at $z=r_{0} e^{i \theta_{0}}$. Consequently, since

$$
\frac{\partial}{\partial \theta} \arg p(z)=\operatorname{Im}\left\{\frac{\partial}{\partial \theta} \ln p(z)\right\}=\operatorname{Im}\left\{i z \frac{p^{\prime}(z)}{p(z)}\right\}=\operatorname{Re}\left\{\frac{z p^{\prime}(z)}{p(z)}\right\},
$$

then

$$
\operatorname{Re}\left\{z p^{\prime}(z) / p(z)\right\}=0
$$

at $z=r_{0} e^{i \theta_{0}}$. We use (1), (2), (3) and (4) to get

$$
\operatorname{Re}\left\{I\left(\alpha, f\left(z_{0}\right)\right)\right\}=\operatorname{Re}\left\{(1-\alpha) p\left(z_{0}\right)+\alpha\left(1+\frac{z_{0} p^{\prime}\left(z_{0}\right)}{p\left(z_{0}\right)}\right)\right\}=\alpha .
$$

Thus if $\alpha<0$, then (5) shows that $f \notin H(\alpha)$ which is a contradiction. This completes the proof of Theorem 1 .

3. The radius $r_{\alpha}$. Let $P$ denote the class of regular functions $p(z), p(0)=1$, with positive real part, $\operatorname{Re}\{p(z)\}>0, z \in E$. If $f \in \mathrm{NW}$, then there is a $p(z) \in P$ such that $f^{\prime}(z)=p(z)$. By substitution (1) becomes $I(\alpha, f(z))=\varphi_{\alpha}\left(p, z p^{\prime}\right)=(1-\alpha) p(z)+\alpha\left(1+z p^{\prime}(z) / p(z)\right)$. Let

$$
Q_{\alpha}(r)=\min _{p \in P} \min _{|z|=r<1} \operatorname{Re}\left\{(1-\alpha) p(z)+\alpha\left(1+z p^{\prime}(z) / p(z)\right)\right\} .
$$

Hence, the problem of finding the largest $r_{\alpha}$, for fixed $\alpha$, such that for each $f \in \mathrm{NW}$ and for each $z,|z|<r_{\alpha}$ we have $\operatorname{Re}\{I(\alpha, f(z))\}>0$ is equivalent to finding the smallest positive root of $Q_{\alpha}(r)=0$, where $Q_{\alpha}(r)$ is given by (6). To find $r_{\alpha}$, we make use of a theorem due to V. A. ZMOROvIC [5]. 
Theorem A (ZMorovic). Let $\Psi(w, W)=M(w)+N(w) W$, where $M(w)$ and $N(w)$ are defined and are finite in the half plane $\operatorname{Re}\{w\}>0$. We set

$$
\begin{aligned}
w & =\lambda_{1}\left(1+z_{1}\right) /\left(1-z_{1}\right)+\lambda_{2}\left(1+z_{2}\right) /\left(1-z_{2}\right), \\
W & =\lambda_{1} 2 z_{1} /\left(1-z_{1}\right)^{2}+\lambda_{2} 2 z_{2} /\left(1-z_{2}\right)^{2},
\end{aligned}
$$

where $z_{1}$ and $z_{2}$ are points on the circumference $|z|=r<1, \lambda_{k} \geqslant 0$ $(k=1,2), \lambda_{1}+\lambda_{2}=1$. Then the function $\Psi(w, W)$ can be put in the form

$$
\Psi(w, W)=M(w)+\frac{1}{2}\left(w^{2}-1\right) N(w)+\frac{1}{2}\left(\varrho^{2}-\varrho_{0}^{2}\right) N(w) e^{2 i v},
$$

where

$$
\left(1+z_{k}\right) /\left(1-z_{k}\right)=a+\varrho \exp i \psi_{k}(k=1,2) \text {, }
$$

$$
w=a+\varrho_{0} \exp i \psi_{0} \quad\left(0 \leqslant \varrho_{0} \leqslant \varrho\right), \quad\left|z_{1}\right|=\left|z_{2}\right|=r,
$$

$a=\left(1+r^{2}\right) /\left(1-r^{2}\right), \quad \varrho=2 r /\left(1-r^{2}\right), \quad \exp i \psi=i \exp \left[\frac{1}{2}\left(\psi_{1}+\psi_{2}\right)\right]$.

Also for a fixed $w$ in the circle $|w-a|<\varrho$, the angle $2 \psi$ in the above formula can take all values from $[0,2 \pi]$, and hence

$$
\begin{gathered}
\min \operatorname{Re}\left\{\Psi(w, W) \equiv \Psi_{\varrho}(w)=\right. \\
=\operatorname{Re}\left\{M(w)+\frac{1}{2}\left(w^{2}-1\right) N(w)\right\}-\frac{1}{2}|N(w)|\left(\varrho^{2}-\varrho_{0}^{2}\right) .
\end{gathered}
$$

This minimum is reached when

$$
\exp [i(2 \psi+\arg N(w))]=-1 .
$$

We shall need the following result:

Lemma 1. Let

$$
\min \operatorname{Re}\{\Psi(w, W)\} \equiv \Psi_{g}(w)=
$$

$$
=\operatorname{Re}\left\{(1-\alpha) w+\alpha+(\alpha / 2)\left(w^{2}-1\right) / w\right\}-(|\alpha| / 2)\left(\left(\varrho^{2}-\varrho_{0}^{2}\right) /|w|\right),
$$

where $\operatorname{Re}\{w\}>0$. Then the $\min \Psi_{\varrho}(w)$ in the circle $|w-a|=\varrho_{0} \leqslant \varrho$ is reached i) on the diameter if $\alpha \geqslant 0$ and $r \in(0,1)$, and ii) on the diameter if $\alpha<0$ and $r \in(0, \tilde{r})$, where

$$
\tilde{r}=\left(\frac{1+\alpha+(\alpha(\alpha-2))^{1 / 2}}{1-3 \alpha+(\alpha(\alpha-2))^{1 / 2}}\right)^{1 / 2} .
$$

Proof: (i) For $\alpha \geqslant 0$. Let $w=a+\xi+i \eta, R^{2}=|w|^{2}=(a+\xi)^{2}+$ $+\eta^{2}$, where $a, \varrho$, and $\varrho_{0}$ are defined in Theorem A. Then from (9) we get

$\Psi_{\varrho}(w) \equiv \psi_{\varrho}(\xi, \eta)=(a+\xi)\left(1+\frac{\alpha}{2}-\frac{\alpha}{2 R^{2}}\right)-\frac{\alpha}{2} \cdot \frac{\varrho^{2}-\xi^{2}-\eta^{2}}{R}+\alpha$. 
The above yields

$$
\partial \psi_{\varrho}(\xi, \eta) / \partial \eta=\eta \alpha R^{-4} / 2\left[2(a+\xi)+\left(\varrho^{2}-\xi^{2}-\eta^{2}\right) R+2 R^{3}\right] .
$$

The expression in the square brackets is positive; consequently for each fixed $\xi$, the nonnegative minimum of $\psi_{\ell}(\xi, \eta)$ is achieved at $\eta=0$. It follows that the minimum in the circle $\xi^{2}+\eta^{2} \leqslant Q^{2}$ is also reached on the diamenter $\eta=0$.

(ii) For $\alpha<0$. Let $w=R \exp i \varphi$. Then (9) becomes

$$
\begin{array}{r}
\psi_{\varrho}(w) \equiv L(R, \varphi)=\left[(1-\alpha / 2) R-(\alpha / 2) R^{-1}-|\alpha| a\right] \cos \varphi+\alpha+ \\
+(|\alpha| / 2)\left(R+R^{-1}\right) .
\end{array}
$$

From (11) one can conclude only that the minimum of $L(R, \varphi)$ on any arc $R=$ constant inside the circle $|w-a| \leqslant \varrho$ is reached either when $\varphi=0$ or at the end points of this are which are located on the circumference $\varrho=\varrho_{0}$. But by setting $\varrho=\varrho_{0}$ in (9) we get

where

$$
L(R, \varphi)=\left[(1-\alpha / 2) R-\alpha / 2 R^{-1}\right] \cos \varphi+\alpha,
$$

$$
R^{2}-2 a R \cos \varphi+1=0, \quad R \in[a-\varrho, a+\varrho] .
$$

Eliminating $\varphi$ between (12) and (13) we get

$$
L(R)=(1 / 2 a)\left[R^{2}+1\right]-(\alpha / 4 a)\left[R+R^{-1}\right]^{2}+\alpha .
$$

We now show the minimum of $L(R)$ cannot be zero in $[a-\varrho, a+\varrho]$ if $r<\tilde{r}$ where $\tilde{r}$ is given by (10). Consequently, the minimum of $\Psi_{\varrho}(w)$ may vanish on the diameter $\varphi=0$, if $r<\tilde{r}$, which is our lemma. With $a^{2}=\varrho^{2}+1,(14)$ yields

$$
L(a-\varrho)=a-\varrho+\alpha(1-a)
$$

and

$$
L(a+\varrho)=a+\varrho+\alpha(1-a) .
$$

Since $\alpha<0, a>1$ and $a-\varrho>0$, then $L(a-\varrho)>0$ and $L(a+\varrho)>0$. On the other hand, from (14) we obtain

$$
d L(R) / d R=R / a-(\alpha / 2 a)\left[R+R^{-1}\right]\left[1-R^{2}\right]=0
$$

if $R=[\alpha /(\alpha-2)]^{\frac{1}{2}}$. However, $d^{2} L(R) / d R^{2}>0$ at $R=[\alpha /(\alpha-2)]^{\frac{1}{2}}$. This shows that the minimum of the continuous function $L(R)$, if it is not attended at an end point, it must be attended in $(a-\varrho, a+\varrho)$ at $R=[\alpha /(\alpha-2)]^{\ddagger}$. Direct computations show 


$$
\begin{aligned}
& L\left(\left(\frac{\alpha}{\alpha-2}\right)^{\frac{1}{2}}\right)= \\
& =\frac{1}{4 a}\left[\left[4 \alpha \alpha-2 \alpha+2+2 \sqrt{\frac{\alpha}{\alpha-2}}-\alpha \sqrt{\frac{\alpha-2}{\alpha}}-\alpha \sqrt{\frac{\alpha}{\alpha-2}}\right]\right. \\
& =\frac{1}{4 a}\left[4 \alpha a-2 \alpha+2+(2-\alpha) \sqrt{\frac{\alpha}{\alpha-2}}+\sqrt{\alpha(a-2)}\right] \\
& =(1 / 4 a)[4 \alpha a-2 \alpha+2+2 \sqrt{\alpha(\alpha-2)}] \text {. }
\end{aligned}
$$

Hence $L\left([\alpha /(\alpha-2)]^{x}\right)>0$ provided

$$
a<(\alpha-1-\sqrt{\alpha(\alpha-2)}) / 2 \alpha .
$$

Since $a=\left(1+r^{2}\right) /\left(1-r^{2}\right)$, then the above condition is equivalent to (10). Thus $L(R)>0$ in $[a-\varrho, a+\varrho]$ provided $r<\tilde{r}$ and $\tilde{r}$ is as given by (10). This completes the proof of Lemma 1 .

The following theorem describes $r_{\alpha}$.

Theorem 2. Let $f(z)$ be in the class of normalized regular functions with Re $\left\{f^{\prime}(z)\right\}>0$ for $z \in E$. Then $f \in H(\alpha)$ in $|z|=r<r_{\alpha}$ where

i) $r_{\alpha}=(1+\sqrt{2 a})^{-1}, \quad \alpha \geqslant 0$,

ii) $r_{\alpha}=\sqrt{(1-\alpha-\sqrt{\alpha(\alpha-1)}) /(1-\alpha)}, \quad \alpha<0$.

All results are sharp.

Proof: In (3) it is shown that the minimum in (6) is attained by a function of the form

$$
P(z)=\lambda_{1} \frac{1+z e^{-i \theta_{1}}}{1-z e^{-i \theta_{1}}}+\lambda_{2} \frac{1+z e^{-i \theta_{2}}}{1-z e^{-i \theta_{2}}},
$$

where $\theta_{1}, \theta_{2}$ are arbitraxy real constants in $[0,2 \pi]$ and where $\lambda_{1}, \lambda_{2}$ are nonnegative numbers satisfying $\lambda_{1}+\lambda_{2}=1$. We may, therefore, apply $(7)$ to $(6)$ with $p(z)=w(z), M(w)=(1-\alpha) w(z)+\alpha$, and $N(w)=\alpha / w(z)$ to get

$\min \operatorname{Re}\{\psi(w, W)\} \equiv \psi_{\varrho}(w)=$

$$
=\operatorname{Re}\left\{(1-\alpha) w+\alpha+(\alpha / 2)\left(w^{2}-1 / w\right)\right\}-(|\alpha| / 2)\left(\varrho^{2}-\varrho_{0}^{2} \| w \mid\right) .
$$

Note that $\left(9^{\prime}\right)$ is $(9)$ of Lemma 2. Let $w=R \exp i \varphi$. Then $\left(9^{\prime}\right)$ becomes

$$
\begin{aligned}
& \psi_{e}(w)=L(R, \varphi)= \\
& \quad=\left[(1-(\alpha / 2)) R-(\alpha / 2) R^{-1}-|\alpha| a\right] \cos \varphi+\alpha+(|\alpha| / 2)\left(R+R^{\prime}\right)
\end{aligned}
$$


(i) Let $\alpha>0$. By Lemma 1, part (i) the minimum of $\psi_{Q}(w)$ in the circle $|w-a|<\varrho$ is reached on the diameter of this circle $\varphi=0$. In view of this, put $\varphi=0$ in $\left(11^{\prime}\right)$

$$
L(R, 0) \equiv L(R)=R-\alpha a+\alpha .
$$

The minimum of $L(R)$ is at the end of the diameter $R=a-\varrho$. If we set

$$
Q_{\alpha}(r)=L(a-\varrho)=a-\varrho-\alpha a+\alpha
$$

with $a=\left(1+r^{2}\right) /\left(1-r^{2}\right)$, then the least positive root of $Q_{\alpha}(r)=0$ is $r_{\alpha}=1 /(1+\sqrt{2 \alpha})$ this completes our proof of part (i) of the present theorem.

(ii) Let $\alpha<0$. Again Lemma 1, part (ii) shows that the minimum of $\psi_{\varrho}(w)$ is on the diameter $\varphi=0$ for $r<\tilde{r}$, and $\tilde{r}$ is given by (10). However, direct calculations show that $r_{\alpha}<\tilde{r}$ if $r_{\alpha}$ is given by part (ii) of Theorem 2. Therefore, if we set $\varphi=0$ in $\left(11^{\prime}\right), \alpha<0$ we get $l(R) \equiv L(R, 0)=(1-\alpha) R-\alpha R^{-1}+\alpha(1+a), \quad R \in[a-\varrho, a+\varrho]$. Then it follows that

$$
d l(R) / d R=(1-\alpha)+\alpha R^{-2}=0
$$

for $R^{2}=\alpha /(\alpha-1)$, or $R=\sqrt{\alpha /(\alpha-1)} \equiv R_{0}$. It is clear that $R_{0}=$ $=\sqrt{\alpha /(\alpha-1)}<1<a+\varrho$ but $R_{0}$ is not necessarily greater than $a-\varrho$. Hence the minimum is either attained at $R_{0}=\sqrt{\alpha /(\alpha-1)}$ or at $R_{1} \equiv a-\varrho$. For the latter case, we find

$$
l\left(R_{1}\right)=(1-\alpha)(a-\varrho)-\alpha(a-\varrho)^{-1}+\alpha(1+a)
$$

does not vanish for real $r$. The other alternative is

$$
Q_{\alpha}(r) \equiv l\left(R_{0}\right)=2 \sqrt{\alpha(\alpha-1)}+\alpha(1+a)
$$

whose smallest positive zero is $r_{\alpha}=\sqrt{(1-\alpha-\sqrt{\alpha(\alpha-1)}) /(1-\alpha)}$. Our proof of the theorem is now complete.

We now determine the extremal functions $f_{0}(z)$. We remark that as a consequence of (8) the minimum of $\left(9^{\prime}\right)$ is reached when the point $w(|w-a|<\varrho)$ is fixed, and the chord passing through it and through the points $a+\varrho \exp \psi_{k}(k=1,2)$ is perpendicular to the vector $\exp (i \varphi / 2)$, where $w=R \exp i p$. Taking this into account, as well as the fact that the minimum of $\psi_{\varrho}(w)$ is realized at an end point of the diameter when $\alpha \geqslant 0$, we conclude that $p(z)$ of (15) 
should be taken in the form $p(z)=(1+z) /(1-z)$. Hence for $\alpha \geqslant 0$, the extremal function is

$$
f_{0}(z)=-\ln (1-z)-z
$$

which realizes part (i) of Theorem 2 at $z=-r$. For $\alpha<0$, the minimum is reached at a point of the diameter (not an end point) and thus $p(z)$ should in this case be taken in the form.

$$
p(z)=\frac{1}{2} \frac{1+z e^{-i \theta}}{1-z e^{-i \theta}}+\frac{1}{2} \frac{1+z e^{i \theta}}{1-z e^{i \theta}},
$$

where $\theta$ is given by the relation

$$
R_{0}=\sqrt{\alpha /(\alpha-1)}=\operatorname{Re}\{p(z)\}=\left(1-r_{\alpha}^{2}\right)\left(1-2 r_{\alpha} \cos \theta+r_{\alpha}^{2}\right)^{-1}
$$

and $r_{\alpha}$ is given by (ii) of the Theorem 2. This shows

$$
f_{0}(z)=-\left[e^{i \theta} \ln \left(1-z e^{-i \theta}\right)+e^{-i \theta} \ln \left(1-z e^{i \theta}\right)+z\right] .
$$

4. A coefficient inequality for functions in $H(\alpha)$. In this section we obtain some coefficient properties for functions in $H(\alpha)$. We show the following theorem.

Theorem 3. If $f(z)=z+\sum_{n=2}^{\infty} a_{n} z^{n}$ is in $H(\alpha)$ and if $\mu$ is an arbitrary complex number, then

$$
|1+\alpha|\left|a_{3}-\mu a_{2}^{2}\right| \leqslant \frac{2}{3} \max \left[1,\left|1+2 \alpha-\frac{3}{2} \mu(1+\alpha)\right|\right] .
$$

Proof: If $f(z) \in H(\alpha)$, then there exists a regular function. $w(z)=\sum_{n=1}^{\infty} c_{n} z^{n}$ such that $|w(z)|<1$ in $E$ and

$$
(1-\alpha) f^{\prime}(z)+\alpha\left(1+z f^{\prime \prime}(z) / f^{\prime}(z)\right)=(1+w(z)) /(1-w(z)) .
$$

Now by expanding (18) and equating coefficients we have

$$
a_{2}=c_{1}
$$

and

$$
3(1+\alpha) \alpha_{3}-4 \alpha a_{2}^{2}=2\left(c_{2}+c_{1}^{2}\right) .
$$

We may assume $\alpha \neq-1$. From (19) and (20) we get

$$
\left|a_{3}-\mu a_{2}^{2}\right|=(2 / 3|1+\alpha|) c_{2}-\left(1+2 \alpha-\frac{3}{2} \mu(1+\alpha)\right) c_{1}^{2} \mid \text {. }
$$

A result due to $\mathrm{K}$ mogH and Merkes [1] shows that $\left|c_{2}-v c_{1}\right| \leqslant$ $\leqslant \max [1,|v|]$ for arbitrary complex number $v$. We apply this result 
to the right hand side of (21) with $\nu=1+2 \alpha-\frac{3}{2} \mu(1+\alpha)$ to obtain (17). This completes Theorem 3.

Remarks. i) For $\alpha=1,(17)$ reduces to

$$
\left|a_{3}-\mu a_{2}^{2}\right| \leqslant \max \left[\frac{1}{3},|\mu-1|\right]
$$

which is a result of Keogrt and Menkes [1].

ii) For $\alpha=0,(17)$ reduces to

$$
\left|a_{3}-\mu a_{2}^{2}\right| \leqslant \max \left[\frac{2}{3},\left|\mu-\frac{2}{3}\right|\right] \text {. }
$$

Corollary. If $f(z) \in H(\alpha)$, then

$$
\left|a_{2}\right| \leqslant 1
$$

and $\quad|1+\alpha|\left|a_{3}\right| \leqslant \begin{cases}\frac{2}{3} & \text { if }-1 \leqslant \alpha \leqslant 0 \\ \frac{2}{3}|1+2 \alpha| & \text { if }|1+2 \alpha|>1 .\end{cases}$

Proof: The inequalities in (22) and (23) follow directly from (19) and (21), respectively.

\section{References}

[1] Kroger, F. R., and E. P. MERKFs : A coefficient inequality for certain classes of analytio functions. Proc. Amer. Math. Soc. 20, 8-12 (1969).

[2] Nostriro, K.: On the theory of schlicht functions. J. Fac. Sci. Hokkaido Univ. Ser. 1, 2, 29-155 (1934-1935).

[3] RoBertson, M. S.: Extremal problems for analytic funotions with positive real part and applications. Trans. Amer, Math. Soc. 106, 236-253 (1963).

[4] WARschawskI, S.: On the higher derivatives at the boundary in conformal mappings. Trans. Amer. Math. Soc. 38, 310-340 (1935).

[5] Zuronovro, V. A.: On bounds of eonvexity for starlike functions of order $\alpha$ in the circle $|z|<1$ and in the circular region $0<|z|<1$. Amer. Math. Soc. Transl. (2) 80, 203-213 (1969).

H. S. AL-AMrrR

Department of Mathematics Bowling Green State University Bowling Green, OH 43403, USA
M. O. RFADE

Department of Mathematies University of Michigan Ann Arbor, MT 48104, USA 